\title{
Un inserto proustiano in una prosa politica di Giacomo Debenedetti
}

\section{Raffaele De Cesare}

\section{(2) OpenEdition}

1 Journals

\section{Edizione digitale}

URL: http://journals.openedition.org/studifrancesi/32651

DOI: 10.4000/studifrancesi.32651

ISSN: 2421-5856

\section{Editore}

Rosenberg \& Sellier

\section{Edizione cartacea}

Data di pubblicazione: 1 décembre 2005

Paginazione: 510-516

ISSN: 0039-2944

\section{Notizia bibliografica digitale}

Raffaele De Cesare, «Un inserto proustiano in una prosa politica di Giacomo Debenedetti», Studi Francesi [Online], 147 (XLX | III) | 2005, online dal 30 novembre 2015, consultato il 19 avril 2021. URL: http://journals.openedition.org/studifrancesi/32651; DOI: https://doi.org/10.4000/studifrancesi. 32651

\section{(c) (i) (9)}

Studi Francesi è distribuita con Licenza Creative Commons Attribuzione - Non commerciale - Non opere derivate 4.0 Internazionale. 


\section{Un inserto proustiano in una prosa politica di Giacomo Debenedetti}

Insieme a Giovanni Macchia e - sul versante linguistico - a Gianfranco Contini, Giacomo Debenedetti rappresenta una delle tre vette della catena montuosa innalzata, roccia su roccia, da quasi un secolo a questa parte, dall'operosità esegetica proustiana nella nostra Penisola; vetta che non solo domina il paesaggio critico italiano, ma che sovrasta con l'imponenza della sua altezza l'intero territorio europeo ed americano. Onde dispiace - e sia detto senza alcuna tentazione campanilistica - che poco si parli ed ancor meno si discuta delle indagini innovatrici, sottili e luminose, di Debenedetti intorno alla Recherche du temps perdu nei repertori bibliografici, negli «états présents», nei manuali relativi a Proust che hanno recentemente visto la luce, e continuano copiosamente a rivederla, in Francia e nel mondo.

\section{$*$}

All'opera proustiana Giacomo Debenedetti dedicò, fra il 1925 ed il 1965, ben cinque ampi saggi, oltre a numerosi riferimenti in altri scritti: riferimenti non sempre colti al volo per suggerire elementi di somiglianza o di affinità con problemi espressamente trattati, ma veri e propri appigli intellettuali, meditate occasioni per una riflessione critica più generale.

Lettore, fin dall'estate del 1924 dei primi due volumi del romanzo, il Du côté de chez Swann, egli scrisse e pubblicò nell'aprile del 1925 un primo, pioneristico studio, Proust $1925^{1}$. Pioneristico, ma già frutto di una esplorazione attenta, contrassegnata da un vigile distacco dell'intelligenza verso la propria scoperta. In esso, traboccante di entusiasmo per un'opera già giudicata di una straordinaria originalità nel paesaggio narrativo francese, Debenedetti analizzava l'opera proustiana, grazie ad una lettura attenta alle risonanze musicali, agli effetti cromatici, all'assetto stilistico che le erano propri; e di essa lodava la continuità del suo «ininterrotto e quasi fatale fluire», la compattezza della sua ispirazione, la modulazione tonale che animava l'insieme degli episodi narrati, «la vasta sinfonia che fasciava l'intero racconto» «la magia poetica che tutto avvolgeva», «la stupefacente ricchezza di particolari che pullulavano intorno ad ogni argomento toccato», la maestria stilistica che sovraintendeva l'esposizione.

Meno di tre anni dopo, ne «La Rassegna musicale» del $1^{\circ}$ gennaio 1928, Debenedetti, completata la lettura della Recherche e dominato così l'intero percorso del romanzo fino all'approdo estremo del Temps retrouvé, forte della propria esperienza e sensibilità musicali, affrontava in Proust e la musica ${ }^{2}$ un tema particolare: quello dell'interesse, del gusto, delle preferenze di Proust per la musica e (anche sulla scorta dei documenti tramandatici da quella parte della Correspondance allora nota) degli aspetti che essa assumeva nei celebri episodi della Reherche. Premesso che il carattere del gusto musicale di Proust è «eclettico» ed «antologico», che il suo culto per la musica non è tanto quello di un tecnico, specialista nella materia, quanto quello di uno scrittore teso a rappresentare l'alone magico che si diffonde in un determinato personaggio, in un preciso momento della sua vita affettiva, il critico orientava il suo discorso verso il problema delle «intermittences du coeur», tema che è la vera, grande

(I) Ora in Saggi, Milano, Mondadori, 1999, pp. 265-282. Tralasciando l'indicazione bibliografica dell'edizione originale, di ognuno di tali saggi sarà fatta referenza a quest'ultima stampa mondadori- ana, detta dei Meridiani, come alla più completa, accurata ed accessibile edizione.

(2) Saggi, ed. cit., pp. 283-299. 
scoperta di Proust. Ed insisteva sulla funzione che la musica, redentrice della «sécheresse de l'âme» e mediatrice delle insorgenze della memoria involontaria, esercitava in tutto il tessuto narrativo del romanzo.

Nell' aprile di questo stesso anno 1928, a Milano, al «Circolo del Convegno», Debenedetti commemorava Proust ${ }^{3}$. Ma più che una commemorazione ufficiale, il suo discorso era un ininterrotto elogio, commosso e sereno ad un tempo, di uno scrittore che il conferenziere definiva «mago notturno e favoloso», «il più cospicuo rappresentante del romanzo contemporaneo». Di lui, Debenedetti celebrava l'opera («eroica intrapresa poetica e umana») e rievocava con partecipe simpatia, la vita. E, nel riassumere le vicende che costituivano la trama della Recherche, dal Du coté de chez Swann al Temps retrouvé, il critico tornava ad insistere sull'invenzione di quelle «intermittences du coeur» che danno ragione di esistere al romanzo, lo giustificano e, sollecitando nell'autore la vocazione artistica, lo scandiscono via via e lo concludono. Anche allo stile proustiano (argomento allora, di appassionate discussioni, il più delle volte ostili). Debenedetti attribuiva qualità di novità e di eccellenza; e lo faceva con espressioni tipiche della sua eleganza e della sua penetrazione di raffinato lettore: «[Proust] è arrivato... a costruirsi un suo stile che vince, persuade e fluidifica le più ostinate e sorde resistenze della materia, che ha le libere e ascensionali audacie, le riflessioni fantastiche e gioiose, i serrati e pur volubili estri canori del più bel gotico che sia fiorito su per le cattedrali di Francia o su per le torri e gli edifici illustri della vecchia Parigi».

Circa venticinque anni dopo, proludendo nel febbraio del 1951 al corso di letteratura italiana moderna e contemporanea all'Università di Messina (insegnamento cui era stato chiamato fin dall'anno prima), Debenedetti tornava nuovamente a Proust con lo scritto Marcel Proust a patti col Diavolo ${ }^{4}$. Questa volta non più per delineare un ritratto a tutto tondo del romanziere, per tratteggiarne la personalità umana e letteraria, ma per sondare un problema dalle incognite complesse e misteriose: la Recherche vista nella prospettiva del romanzo di una crisi esistenziale, penetrato da inibizioni di fondo, la cui altissima resa artistica non poteva essere raggiunta se non attraverso una lotta aspra e sorda. Per rimuovere ogni impedimento dello spirito, per «lacerare il diaframma creatosi tra la propria capacità di esprimersi e il proprio bisogno di esprimersi», l'uomo moderno non ha altro ricorso che una sorta di patto col Diavolo. Grazie ad esso, in cambio della cessione dell'anima in eterno, ottiene «qualche attimo, terribilmente fecondo, di pieno possesso della propria anima. Si fa pronubo di momentanee e generative nozze tra animus e anima, per dirla con gli psicologi di tanto tempo fa e di adesso». Certo, in tale patto diabolico, è giocoforza imbattersi in rischi e subire ricatti. Ma il contraente-uomo può in definitiva vincere l'altro protagonista del mercato ed averne ragione. E' stato il caso di Proust che «... dopo aver subito anche lui, per la sua parte, i ricatti del Diavolo, riesce a sconfiggerlo in una maniera trionfale, innocente e senza doppi sensi: una maniera da finale di sacra rappresentazione». Si innesta qui un sottile gioco di richiami e di rapporti fra la Recherche, il Doktor Faust di Thomas Mann e, parallelamente, le teorie psicologiche di Carl Gustav Jung enunciate, in particolare, nell'opera Psicologia e Alchimia che Debenedetti intesseva con acuta destrezza. Tuttavia, più che seguire il critico in questo itinerario - impervio almeno per quanti come noi, sono ignari delle argomentazioni psicoanalitiche - ci è agevole prendere atto dell'ininterrotta ammirazione di questi per la Recherche: ammirazione affidata a formule squisite. Ne richiamiamo due sole: l'opera proustiana è una «fluida,

(3) Commemorazione di Proust, ora in Saggi, ed. cit., pp. 300-328.
(4) Ora in Saggi, ed. cit., pp. 925-950. 
stupenda, incessante. calligrafia di luce» della durata dell'esistenza; una «sinfonia... tra le più ricche orchestrazioni della parola che siano state create».

L'ultimo contributo di Debenedetti alla esplorazione dell'universo proustiano apparteneva all'ottobre dell'anno successivo, spostava il suo obbiettivo dalla Recherche al Jean Santeuil e si presentava sotto una veste nuova, almeno nella sua diversa realizzazione tecnica5. Da pochi mesi (nel maggio) Bernard de Fallois aveva restituito alla luce e pubblicato il romanzo giovanile di Proust. Sotto forma di Radiorecita, il 10 ottobre 1952, Debenedetti allestì per il Terzo Programma della Radio italiana una trasmissione nella quale le parole di un critico, le interruzioni di una donna intelligente ed intrigante («faiseuse d'embarras», come la definiva l'autore), gli stralci del romanzo citati da alcuni lettori, i dubbi di un pubblico impaziente si accompagnavano ad intermezzi musicali destinati a suggerire emozioni e a ricreare l'atmosfera più consonante alla rievocazione verbale del testo. In questo quadro nuovo (e talora sconcertante) del restauro letterario proustiano, Debenedetti commentava la recentissima edizione del de Fallois, riassumeva compendiosamente il romanzo, coglieva i preannunci ivi contenuti della Recherche, sottolineava le analogie con essa, procedeva ad osservazioni di lettura e ad un giudizio d'insieme. Il quale giudizio si sintetizzava in queste considerazioni: Jean Santeuil non era certamente un capolavoro, ma conteneva pagine abbaglianti che era stato un peccato aver sacrificato nella redazione definitiva della Recherche, e, in ogni caso, costituiva una preziosa testimonianza dei Lebrjabre di Proust.

I cinque saggi ora ricordati non esauriscono l'interesse di Debenedetti per l'opera proustiana. Come già si è accennato, numerosi sono, lungo il percorso letterario di lui, i luoghi in cui la Recherche è diversamente richiamata alla memoria, segnalata, celebrata per uno dei tanti aspetti peculiari della sua essenza narrativa ${ }^{6}$.

Impossibile discutere ciascuno di questi passi e riprodurli qui in esteso. Ma è necessario dire almeno che essi si susseguono dal 1925 al 1965 con sorprendente frequenza. Il $1^{\circ}$ febbraio 1925, in Cauto omaggio a Radiguet ${ }^{7}$, Proust è citato, accanto a Gide ed a Freud fra i «maggiori astri» dell'Olimpo intellettuale europeo. Il 16 febbraio dello stesso anno, in Vera natura dei romanzi di Radiguet ${ }^{8}$, è individuato come autore delle più importanti «scoperte psicologiche». Il 5 maggio 1928, in Per Saba ancora ${ }^{9}$, è identificato come uno degli scrittori fanaticamente amati dal poeta triestino. Nel marzo ed il $1^{\circ}$ settembre 1929, rispettivamente in Lettera a Carocci intorno a Svevo e Schmitz e in l'ultimo Svevo ${ }^{10}$, è chiamato in causa per un possibile parallelo con Svevo (parallelo a cui peraltro Debenedetti non crede). Il 10 genaio 1931, in E parliamo del romanz $0^{11}$, è menzionato fra i «più intelligenti lettori di Flaubert». Nel marzo 1941, in Avvertenza per il 1941 ovvero agitare prima dell' uso ${ }^{12}$, è lodato per aver «avuto il coraggio di lasciarsi vivere, tacendo e soffrendo di tacere, tra tutte le disgregazioni morali dell'estetismo e di una dissipatissima esistenza mondana. Letteralmente egli vi ha perduto la propria vita, vi si è dannato. Ma proprio quella pazienza, quella apparente passività gli permisero di ascendere a uno stato, a una iniziazione, donde gli fu

(5)Radiorecita su «Jean Santeuil», ora in Saggi, ed. cit., pp. 951-984.

(6) E anche da rilevare, a proposito dello stretto legame di Debenedetti con temi e personaggi proustiani, il fatto che, fra il 1926 ed il 1929, le recensioni che egli scriveva per la «Gazzetta del popolo» di Torino sono firmate con lo pseudonimo di Swann

(7) Ora in Saggi, ed. cit., p. 166.

(8) Ora in Saggi, ed. cit., p. 181.

(9) Ora in Saggi, ed. cit., p. 249.

(10) Ora in Saggi, ed. cit., p. 456 e pp. 470-471.

(11) Ora in Saggi, ed. cit., pp. 490-491.

(12) Ora in Saggi, ed. cit., p. 656. 
possibile ritrovare, a traverso i filtri patetici e poetici della memoria, la figura musicale del temps perdu, il filo d'Arianna da dipanare per il labirinto di quel tempo perduto, rintracciandone e ordinandone gli episodi e gli incontri, la servitù e le grandezze». Nell'ottobre-dicembre 1947, in Personaggi e Destino ${ }^{13}$, la duchessa de Guermantes (insieme a don Abbondio, Julien Sorel, padron 'Ntoni, Raskolnikoff, Tonio Kroeger) è ravvisata come uno dei personaggi esemplari della narrativa del nostro Continente; è ricordato l'episodio del ricevimento della marchesa de Saint-Euverte nella Recherche, e - osservazione capitale - è affermato che i personaggi di Proust, di Pirandello, di Joyce, a differenza di quelli della narrativa cosiddetta realistica, sfuggono all'analisi del loro creatore ed a lui, con prepotente autonomia, si ribellano. Nel maggio 1949, in Prefazione 1949. Probabile autobiografia di una generazione (maggio 1949) ${ }^{14}$, Proust è nominato, insieme a Kafka, fra i protagonisti di una generazione tormentata ed insicura. Nel luglio-agosto 1950, nel secondo «capitolo» dedicato all'Alfieri (Nascita delle tragedie $)^{15}$ è posto in risalto quell'aneddoto raccontato nella Vita autobiografica che è una anticipazione dell'insorgenza della memoria involontaria. Nel novembredicembre 1954, in Presagi del Verga ${ }^{16}$ è suggerito un accostamento (in Una peccatrice) fra Pietro Brusio mentre al pianoforte suona a Narcisa Valdieri morente la canzone dei loro giorni felici, e Swann nell'atto di ascoltare «les refrains oubliés du bonheur» musicati da Vinteuil. Il 6 maggio 1956, in La Mancini, l'anima e la danza ${ }^{17}$ è rievocato il proprio entusiasmo giovanile per quegli «scrittori e poeti nuovi» [fra cui Proust] che appagassero anche la nostra infinita nostalgia di beatitudine. e che la facessero finita... coi gesti e le cerimonie». Il 20 luglio 1958, in Buzzati e gli sguardi del «di qua $\gg^{18}$, Proust è nuovamente elencato fra i maggiori romanzieri del secolo XX. Il 21 luglio 1958 e, poi, nel 1963, in Dessi e il Golfo mistico ${ }^{19}$, i racconti del narratore sardo vengono ricondotti (con qualche esagerazione operata da Dessì) nella scia del «metodo di recupero del tempo perduto scoperto da Proust e poi servitogli ad imbastire tutto il suo romanzo». Nel 1959, nella prefazione (Quinta stagione) ad Epigrafe. Ultime poesie di U. Saba ${ }^{20}$, viene nuovamente sottolineata l'ammirazione del poeta di Trieste per La Prisonnière. Nel 1962-1963, nei quaderni preparatori sul tema Joyce e Proust per le lezioni tenute all'Università di Roma (ma pubblicati postumi solo nel 1971 da Renata Debenedetti Orengo ${ }^{21}$, nel corso di una sottile analisi delle «epifanie» di Joyce e delle «intermittences du coeur» di Proust, la Recherche è definita senza riserve «questo capolavoro certo assoluto del nostro secolo e probabilmente di tutta la letteratura a noi nota». Nel 1963-1964, nei successivi quaderni per le lezioni universitarie, Alcuni aspetti del romanzo (pubblicati anch'essi postumi dalla moglie di Debenedetti nel I971) ${ }^{22}$, il posto occupato da Proust continua ad essere nelle prime file e, come sempre, circondato dalla più piena ammirazione. Proust entra in scena, insieme a Joyce, come il capostipite del romanzo nuovo, nato dalle ceneri di quello naturalistico. Dello scrittore irlandese sono di nuovo rammentate le «epifanie»; del narratore francese, le «intermittences du coeur». E di queste seconde è detto che danno vita al «tessuto incantevole, smagliante e doloroso del suo [di Proust] romanzo»; che sono «gli echi stupendi e inesauribili destati incessantemente dal suo stile su una fuga prospettica di piani multipli che si succedono e si richiamano a perdita d'occhio dietro la fluida superficie della sua sinfonia verbale». Pagine di vario tenore vengono poi riferite (a correzione di alcune osservazioni di E. M. Forster sull'bomo fictus) sui

(13) Ora in Saggi, ed. cit., p. 901 e pp. 906-907.

(14) Ora in Saggi, ed. cit., p. 115.

(15) Ora in Saggi, ed. cit., p. 799.

(16) Ora in Saggi, ed. cit., p. 994.

(17) Ora in Saggi, ed. cit., pp. 1139-1140.

(18) Ora in Saggi, ed. cit., p. 1190.
(19) Ora in Saggi, ed. cit., p. 1200.

(20) Ora in Saggi, ed. cit., pp. 1110-1111.

(21) Ora in Saggi, ed. cit., pp. 1327-1353.

(22) Ora in Saggi, ed. cit., pp. 1368-1369; 1370-1378; 1382-1383; 13383-1384; 1387-1389; 1426-1427. 
«prelibati virtuosismi della cuoca Françoise» e, sempre in garbata polemica con le teorie di Forster, sul sonno di Albertine ne La Prisonnière. Successivamente, sono ritratte le fattezze fisiche di taluni personaggi del romanzo (Odette, per esempio) e riprese affermazioni di carattere etico-estetico espresse da Proust nella prefazione ai Tendres Stocks di P. Morand. In ultimo, nel 1965, nella Commemorazione provvisoria del personaggio-uomo ${ }^{23}$, il tema dei rapporti fra Proust, Joyce e il simbolismo e del superamento di quelle posizioni è ancora ribadito; l'atteggiamento di Proust di fronte alle cose del mondo fisico e agli esseri umani è interpretato alla luce di una metafora sartriana della «esplosione» verso se stesso; e captate nella loro segreta essenza, esse vengono recuperate in tal senso; la natura dei personaggi viene trasferita da una entità «omogenea», «compatta» ad una successione di «atomi psicologici o figurativi o figurali, dotati di una straordinaria autonomia, anche se venivano imputati a quel luogo geometrico, o emblema collettivo, o comune denominatore, che sembrava conservare i diritti, le patenti, le investiture del personaggio classico».

Sull'onda, sempre rinnovata, di questo consenso che, con ammirevole continuità, avvince culturalmente e spiritualmente Giacomo Debenedetti all'opera proustiana, non fa meraviglia se un passo della Prefazione, scritta nel 1945 per l'edizione dei Saggi critici. Nuova serie ${ }^{24}$ ci riconduce avvertibilmente ad un episodio de La Prisonnière. Nè appare senza significato se noi lo segnaliamo qui e lo proponiamo ad una particolare attenzione del lettore. Giacchè la somiglianza che si riscontra fra i due testi diventa tanto più eloquente quanto più si rivela inserita in una trama di natura e di intonazione divergenti. Il passo di Debenedetti, infatti, affonda le sue radici in una riflessione di carattere storico-politico, proprio della situazione italiana durante il Fascismo e del malessere che, colto da uno spettatore disincantato, si celava dietro i più fastosi avvenimenti pubblici. Il brano di Proust amaramente commenta (e non per la prima volta) la «sécheresse de l'âme» davanti al dolore ed alla morte, la aridità del cuore che penetrano entro certi ambienti della nobiltà e dell'alta borghesia fran$\operatorname{cesi}^{25}$.

Siamo, per Debenedetti, negli anni intorno al 1937, alla vigilia della seconda guerra mondiale, lo stato della cultura italiana appariva ad uno sguardo superficiale, «nutrito, felice, abbondante di vitalità». Il Fascismo orchestrava propaganda e profondeva denaro per dare alla vita intellettuale dignità e ricchezza. Ma, in realtà, non era che una floridezza illusoria o, perlomeno, una floridezza che investiva solo la «letteratura di corte», prona ai voleri dell'Autorità politica. Ed eccone - quasi un apologo - il commento:

«Strana festa. Chi vi fosse invitato, chi vi giungesse dal di fuori (era il caso del critico) subito riceveva un'impressione indefinibile, senza un dove, senza un perchè, il malessere del «qualcosa-che-non-va». Adesso, dalla prospettiva del poi, si direbbe ch'era come arrivare in una casa per un grande ricevimento con buffé e sonatori, ospiti di riguardo nonchè di eccellente compagnia e ogni sorta di attrattiva. Frattanto nella casa, un'ora prima di quella fissata sul biglietto d'invito, una persona di famiglia

(23) Ora in Saggi, ed. cit., pp. 1283-1322.

(24) Per le Edizioni del Secolo, O.E.T., Roma, Ora in Saggi, ed. cit., pp. 376-377.

(25) Il tema della morte volutamente ignorata, negata, sacrificata davanti alle convenienze mondane per egoismo, aridità di cuore, snobismo è un tema tipicamente proustiano che percorre la Recherche ed abbraccia personaggi di rilievo dal duca de Guermantes a Madame e Monsieur Verdurin (e già affiorava nel Jean Santeuil). Sulla genesi e gli sviluppi di questo tema, che non è qui il caso di esporre, ci limitiamo a rinviare il lettore al nostro saggio Di alcune sinopie balzacchiane nell'opera di Proust di imminente pubblicazione nella Miscellanea in onore di Mario Richter. 
è morta. Ma oramai gli ospiti non si possono più rimandare, i preparativi sono stati fatti, complicati e costosi, e la festa avrà luogo ugualmente. (Si potrà poi sempre dire che la disgrazia è avvenuta all'alba del giorno successivo). E si dà il via all'orchestrina, ermetici servitori circolano coi rinfreschi, si mangia, si beve, si balla, l'atmosfera si fa conviviale, erotica, spiritosa. Nessuno sa, nessuno ha mai saputo, nessuno saprà mai di quel morto. Le donne sono più accese, gli uomini più intraprendenti del necessario, solo qualcuno dei presenti prova un indefinibile disagio. Vorrebbe intonarsi con gli altri, capisce la sconvenienza di quel suo impaccio, di quella sua ritrosìa, di quel suo continuo sottolineare il «qualcosa-che-non-va». Ma che è questo qualcosa? Impossibile dirlo, e chi non è contento, chi non si sente della partita, la colpa è del suo carattere. Lui si sforza, vorrebbe sorridere, e fa una smorfia. C'è qualcosa che non va, c'è quel cadavere all'insaputa di tutti in una stanza della casa in festa - e forse l'han portato ancor caldo nella serra o in una rimessa come usa nei grandi alberghi o nelle cliniche di lusso - c'è la presenza di quel cadavere, tanto più macabra perchè ignota, c'è la «cattiva coscienza» di quel cadavere che si aggira in incognito per la casa in festa dove suonano le musiche e brillano le lampadine elettriche».

Un lettore che abbia qualche familiarità con la Recherche non può fare a meno, a questo punto, di pensare al romanzo proustiano. E di riaprirlo, non tanto per riferirsi all'episodio (in Sodomie et Gomorrhe II) del ballo mascherato a cui partecipa il duca de Guermantes - perfettamente consapevole della morte del cugino d'Osmond, ma non per ciò disposto a sacrificare al luttuoso evento alcuno dei suoi piaceri mondani - quanto, piuttosto, per ritornare su di un avvenimento minore, ma non meno indicativo, occorso durante la grande serata musicale in casa Verdurin, indetta in onore del violinista Morel, e descritta in molte pagine de La Prisonnière. Grazie al prestigio e all'abilità organizzativa del barone de Charlus (che del festeggiato violinista è l'amante) sono convenute le persone più in vista del Faubourg-Saint-Germain, nonchè la stessa ex-regina di Napoli, Maria Sofia di Baviera, allora in esilio a Parigi.

Altro è lo scenario, altro l'apparato, altri sono i personaggi che distinguono le due inquadrature storico-sociali. Ma il fantasma del «morto in casa» (e degli espedienti per nasconderlo), la presenza di un cadavere negato, ignorato, dissimulato, espulso dal pensiero e dagli affetti dei familiari, costituiscono elementi comuni (con qualche variante che non è il caso di rilevare) e rappresentano uno degli snodi della narrazione.

Il lettore provveduto ricorderà. Al termine della esecuzione musicale di Vinteuil e verso la conclusione della serata, Saniette, il mite e maldestro erudito, esprime qualche perplessità sulla qualità dell'interpretazione del violinista Morel rispetto al sentimento generale del settimino eseguito. Infuriato il signor Verdurin, assale, insulta l'interlocutore e lo caccia di casa. Ferito a morte dall'offesa, ingiusta e brutale, Saniette si allontana e cade a terra colpito da un attacco di cuore

«...cinq minutes ne s'étaient pas écoulées depuis l'algarade de M. Verdurin qu'un valet de pied vint prévenir le Patron que M. Saniette était tombé d'une attaque dans la cour de l'hotel. Mais la soirée n'était pas finie».

«Faites-le ramener chez lui, ce ne sera rien» dit le Patron dont l'hôtel «particulier» comme eût dit le directeur de l'hôtel de Balbec, fut assimilé ainsi à ces grands hôtels où on s'empresse de cacher les morts subites pour ne pas effrayer la clientèle et où on cache provisoirement le défunt dans un garde-manger, jusqu'au moment où, eût-il été de son vivant le plus brillant et le plus généreux des hommes, on le fera sortir clandestinement par la porte réservée aux «plongeurs» et aux sauciers ${ }^{26}$.

(26) La Prisonnière, texte établi et annoté par P. E. ROBERT, in $A$ la recherche du temps perdu, édition publiée sous la direction de J.-Y. TADIÉ, Paris,
Gallimard, Bibliothèque de la Pléiade, 1999, III, pp. 769-770. 
Un lettore di sussiego potrà sorridere di commiserazione per la meta - che giudicherà insignificante o trascurabile - a cui è approdato questo nostro vagabondaggio letterario. E, francamente, non oseremmo dargli torto. Ma, ospiti curiosi del laboratorio intellettuale di Giacomo Debenedetti, ci è difficile negare valore all'incontro ora rilevato e mettere in discussione l'opportunità di dargli rilievo. Fra tante testimonianze «in chiaro» della presenza di Proust, questa, nascosta e non rintracciata finora, costituisce una nuova acquisizione. Ed acquisizione tanto più rilevante quanto più la voce di cui abbiamo individuato l'eco proviene da chi, fra i critici italiani del $\mathrm{XX}$ secolo, può considerarsi, per sagacia, per gusto e per eleganza, un maestro di singolare autorità. 